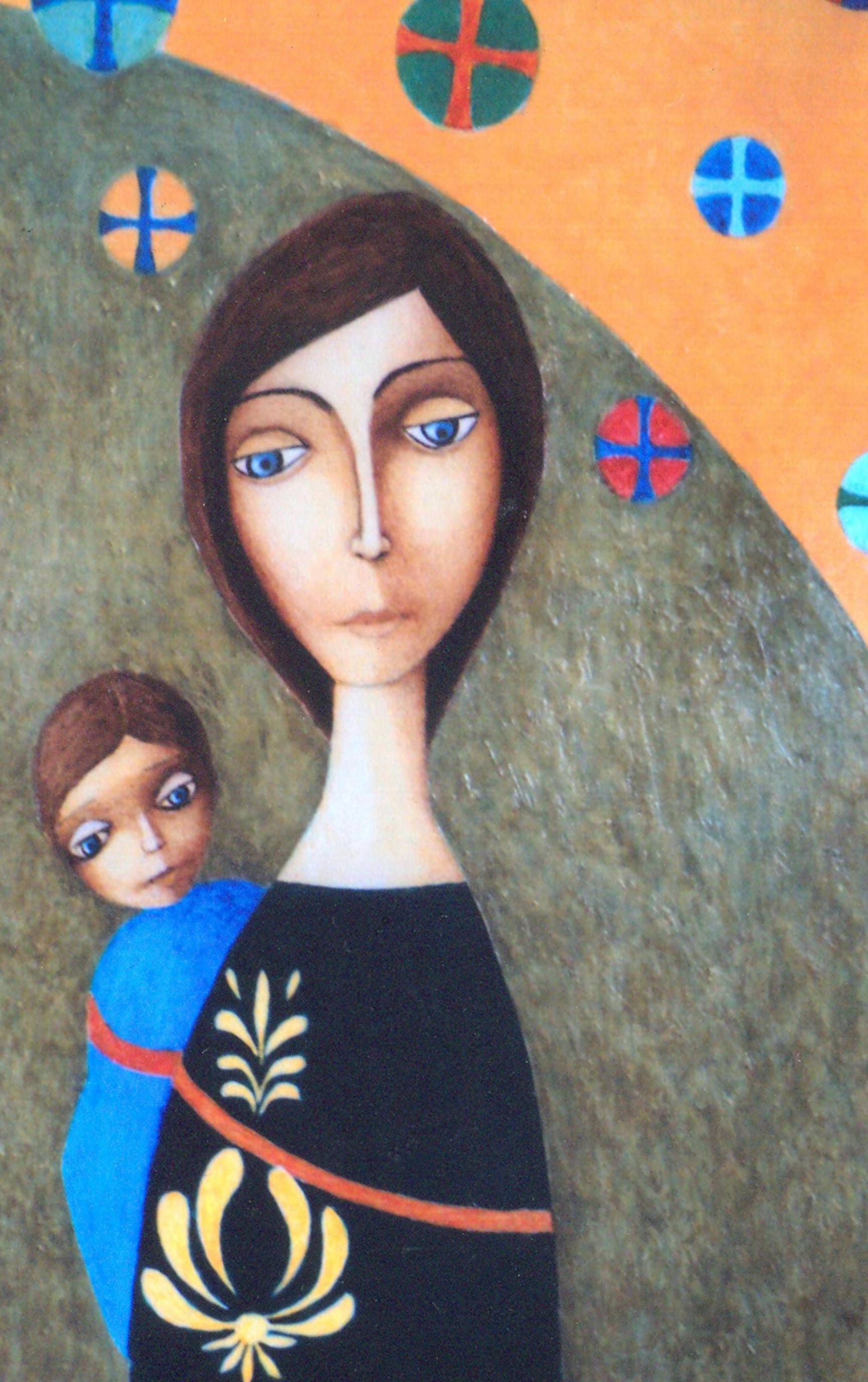




\title{
3 Mortalidad hospitalaria y años de vida potencialmente perdidos en el Hospital San Felipe, año 2014
}

\author{
María Guadalupe Romero-A. * , Ingrid Carolina Garay-R. **
}

RESUMEN. Honduras es un país de ingreso medio que se encuentra en el proceso de transición epidemiológica y una de las formas de constatarlo es a través de los datos de mortalidad hospitalaria basados en el registro de las causas de muerte en los certificados de defunción. Una de las metodologías de medición de la mortalidad hospitalaria es a través de los años de vida potencialmente perdidos propuesto desde 1978 como indicador de salud del estado de una población este indicador cuantifica las muertes prematuras y puede ser utilizado para determinar las prioridades en salud pública. El estudio es retrospectivo, transversal realizado en el Hospital San Felipe de la ciudad de Tegucigalpa, Honduras, centro nacional de referencia y centro nacional de cáncer del país, cuyos datos demuestran que a partir de la causa básica de muerte. El sexo femenino es el que cuantifica el mayor número de años de vida potencialmente perdidos ocasionados por tumores, las septicemias, las afecciones perinatales y las enfermedades del sistema circulatorio. En el caso de los hombres, la cuantificación de los años de vida potencialmente perdidos están relacionados con la diabetes no insulinodependientes, las enfermedades del sistema respiratorio y del aparato digestivo.

Palabra claves: causa básica de muerte, muerte prematura, años de vida potencialmente perdidos.

ABSTRACT. Honduras is a middle-income country that is in the process of epidemiological transition and one of the ways to verify this is through the hospital mortality data whose data are based on the registration of causes of death on death certificates. One of the methods of measurement of hospital mortality by years of potential life lost from 1978 proposed as an indicator of health status of a population, quantifies premature deaths, and can be used to determine the priorities in public health. It is a retrospective cross-sectional study conducted at the San Felipe Hospital in Tegucigalpa, Honduras, national reference center and national cancer center in the country, whose data show that from the underlying cause of death, the female is the quantifying the greatest number of years of potential life lost caused by tumors, sepsis, perinatal disorders and diseases of thecirculatory system. In the case of men, the quantification of the disability-adjusted life year are related to non-insulin dependent diabetes, diseases of the respiratory system and digestive system.

Keywords: basic cause of death, premature death, disability-adjusted life year.

\section{Introducción}

La medición y el análisis de la mortalidad, suscitan el interés de múltiples disciplinas o campos de acción, entre ellos, los sectores de la salud, la demografía, la seguridad social y las políticas sociales en general. Este amplio interés obedece a que la vida constituye el bien más preciado para todos y por ello se realiza el esfuerzo de evitar la muerte y disminuir en la mayor medida posible su incidencia individual y social.

Se agrega que la mortalidad, es un indicador de la situación de salud y también de las condiciones de vida de la población en una amplia variedad de aspectos (CEPAL, 2007). La estadística de causa de muerte sobre las enfermedades asociadas a la causa básica de defunción, constituyen una de las fuentes más importantes en el campo de la salud, así como una medición real del estado de salud de la población según sexo y edad.

Analizando la evolución temporal en el número de defunciones se pueden valorar los resultados obtenidos por las políticas de salud y por las mejoras en los hábitos y estilos de vida de hombres y mujeres (INE, 2014).

Las causas de la mortalidad hospitalaria dependen de las características del hospital, de su complejidad, la

Recibido en abril 2015 / Aceptado y version final en julio 2015

* Médico y Cirujano. Máster en Salud Pública, Máster en Administración de Servicios de Salud. PhD,mgromeroa@yahoo.com

** Ingeniera en Ciencias Ambientales. Máster en Ciencias, Tecnología y Gestión del Agua. PhD, icgaray@usal.es 
importancia de cada uno de los servicios, la prevalencia de las enfermedades en el ingreso al hospital y la gravedad de las mismas. Existen diferencias entre los hospitales privados que atienden a pacientes con condiciones socioeconómicas generalmente similares y los públicos, donde existe mayor número de ingresos en los cuales los enfermos provienen de sectores con diferentes condiciones socioeconómicas y culturales.

En el mundo, la prevalencia de enfermedades en los ingresos hospitalarios puede no coincidir con las de causas de mortalidad, tal es el caso de los hospitales que tienen maternidades, donde la mayor causa de ingresos es por nacimientos e influye poco sobre la mortalidad hospitalaria, por el contrario, las neoplasias son una de las causas prevalentes de mortalidad, especialmente en los países desarrollados aunque no de ingreso hospitalario. Es importante mencionar que la frecuencia de causas de muerte puede no estar relacionada con el número de ingresos por esas causas si no por la letalidad de las mismas (accidentes cerebrovasculares, estadios avanzados de neoplasias malignas, infecciones graves en inmunodeprimidos, insuficiencia coronaria) (Miceli, 2013).

El presente trabajo realiza una revisión de las muertes hospitalarias acaecidas en el año 2014 en el Hospital San Felipe, hospital público de referencia nacional en el cual se realiza una descripción de la causa básica de muerte, las tasas netas y brutas de mortalidad por cada uno de los servicios y los años potenciales de vida perdidos por grandes grupos de causas de muerte.

\section{Aspectos conceptuales}

Se describen a continuación algunos conceptos que se utilizaron en el desarrollo de la investigación

\section{- Defunción}

Es el fallecimiento de toda persona viva independiente de todas las horas que haya vivido.

\section{- Causa de muerte}

Se entiende por causa de defunción, el conjunto de todas las enfermedades, estados morbosos o lesiones que produjeron la muerte o que contribuyeron a ella y las circunstancias del accidente o de la violencia que produjo dichas lesiones.

\section{- Causa básica de defunción}

Enfermedad o lesión que inició la cadena de acontecimientos patológicos que condujeron directamente a la muerte o las circunstancias del accidente o violencia que produjo la lesión fatal. De las cuatro causas de defunción que deben figurar y están informadas en el boletín estadístico se selecciona para ser codificada la que se denomina causa básica que generalmente coincide con la inicial o fundamental y solo en los casos dudosos se recurre para su determinación a las reglas de selección establecidas por la Organización Mundial de la Salud en su Clasificación Internacional de Enfermedades (CIE) (INE, 2014).

\section{- Clasificación internacional de enfermedades 10}

Es un sistema de categorías a las cuales se les asignan entidades morbosas de acuerdo a criterios establecidos. Permite el registro sistemático, al análisis, la interpretación y la comparación de los datos de mortalidad y morbilidad recolectados en diferentes países o áreas y en diferentes épocas. La CIE-10 es un sistema de clasificación de ejes variables cuyo esquema debe servir a todos los propósitos epidemiológicos. Utiliza un código alfanumérico con una letra en la primera posición y números en la segunda, tercera y cuarta posición. El cuarto carácter sigue a un punto decimal, los códigos posibles van por lo tanto de A00.0 a Z99.9 (México.CEMECE, 2009).

\section{- Mortalidad Hospitalaria Bruta y Neta}

Expresa la calidad de la atención médica a nivel hospitalario e intenta discriminarla de la calidad de los servicios de atención ambulatoria. La mortalidad hospitalaria bruta o total expresa el riesgo de morir con respecto al total de egresos acontecidos, la mortalidad neta tiene idéntico propósito, pero circunscribiendo este riesgo a los ingresados de 48 horas o más. El cálculo se realiza a través del cociente del número de defunciones 
hospitalarias totales (tasa bruta) de 48 horas y más de hospitalización (tasa neta) y el número total de egresos para un hospital y período dado multiplicado por 100 . Suele desagregarse este indicador para los diferentes servicios o salas de atención. El tipo de indicador es: Tasa (Ministerio de Salud Pública, La Habana, Cuba, 2010).

\section{- Mortalidad prematura. Años potenciales de vida perdidos}

Son los años que una persona deja de vivir si fallece a una edad que no es la habitual de defunción fijada teóricamente para ese colectivo. Es una medida de la mortalidad que teóricamente se podría evitar (INE, 2014) (OPS B. E., 2003).

Los años de vida potencialmente perdidos es una forma de medición de la carga de una enfermedad conceptualizándose así a la medida de pérdidas de salud que para una población representan las consecuencias mortales y no mortales de las diferentes enfermedades y lesiones. La carga de enfermedad atribuible a una enfermedad concreta se mide por un lado con su frecuencia y por otro lado, a partir de las consecuencias mortales y discapacitantes que origine (Velásquez V, USAID/PERÚ, \& PRAES, 2006).

Un objetivo principal del trabajo de salud pública es aumentar la esperanza de vida de las poblaciones en las mejores condiciones de salud posible. Los años potenciales de vida perdidos es una de las medidas del impacto relativo de varias enfermedades y problemas de salud en la sociedad que ilustran sobre las pérdidas que sufre la sociedad como consecuencia de la muerte de personas jóvenes o de fallecimientos prematuros. Se considera que una muerte es prematura cuando ocurre antes de cierta edad predeterminada que corresponde, por ejemplo a la esperanza de vida al nacer en una población estudiada. Considerar la edad a la cual mueren las personas y no solo el evento mismo de la muerte permite asignar un peso diferente a las muertes que ocurren a diferentes momentos de la vida (Marlow, 1995).

El supuesto en el que se basan los años potenciales de vida perdidos es que cuando más "prematura" es la muerte (más joven se muera), mayor es la perdida de vida. Este indicador tiene por objeto dar una visión amplia de la importancia relativa de las causas más relevantes de mortalidad prematura y su uso fundamental es en la planificación, investigación y definición de prioridades en salud (OPS B. E., 2003) (Martínez Sánchez, Ramos Aceitero, Alvarez Díaz, \& Rojas Fernández, 2013). Los años de vida perdidos tratan de determinar cuántos años más deberían haber vivido las personas que fallecen (Arriaga, 1994).

Se calculan a partir de la diferencia entre la edad de ocurrencia real de la muerte de un individuo y la edad teórica de defunción de éste (OPS, 1986). Esta edad teórica varía: el Center for Diseases Control en Atlanta, Estados Unidos (CDC), estableció una edad de defunción de 65 años para ambos sexos. Otros trabajos la escogieron de acuerdo a la expectativa de vida de cada país. Sin embargo, ambos métodos impiden la comparación de los datos en el contexto internacional. Debido a lo anterior, Murray (1994) decidió utilizar en el Estudio Global de Carga de Enfermedad (Global Bourden of Diseases), un límite único, proveniente de la Tabla de vida modelo West, nivel 26, modificada, que establece una edad teórica de defunción de 80 años para los hombres y de 82.5 años para las mujeres, esto ocurrió cuando esta era la máxima expectativa de vida en el mundo (Japón, 1990).

\section{Metodología}

Se trata de un estudio descriptivo, transversal, retrospectivo en el cual se recogió la información de las muertes acaecidas en el año 2014 en el Hospital San Felipe. Los datos fueron obtenidos de los expedientes de los pacientes que fallecieron en ese período, haciéndose revisión de la hoja de egreso, del certificado de defunción y de la epicrisis (resumen). Estos incluyeron: sexo, edad, procedencia, causa primaria de muerte (de acuerdo a Clasificación Internacional 10). Para el presente trabajo se tomó como dato base, la causa básica de muerte que aparece en la hoja de egreso.

Con los datos se construyeron tablas de frecuencia de mortalidad para ambos sexos y por grupos de edad: menor 
de 1 año; de 1-4 años; de 5-14 años; de 15-44 años; de 45-59 años; de 60 a 70 años y de 71 y más. Se realizaron cálculo de medidas de tendencia central y de desviación con el paquete SPSS versión 21.

Para el cálculo de la mortalidad neta, se utilizó la fórmula: No. de fallecidos en el servicio o en el Hospital de + de 48 horas/ Total de egresos en el servicio o en el Hospital X 100

Para el cálculo de la Mortalidad Bruta, se utilizó la fórmula: No. de fallecidos en el servicio o en el Hospital/ Total de egresos en el servicio o en el hospital X 100

Para el cálculo de los APVP, la fórmula que se utilizó fue:

$\mathrm{L}$

$\mathrm{AVPP}=\Sigma(\mathrm{L}-\mathrm{Xi})$

$\mathrm{x}=0$

Donde $\mathrm{L}=$ es el límite potencial de la vida (80.0 para hombres y 82.5 para mujeres).

$\mathrm{X}=$ es la edad de defunción del sujeto.

El uso de la esperanza de vida al nacer como valor límite de edad, se aplicó para el cálculo de los AVPP ajustándose al perfil poblacional del país para el año 2013. El problema que presenta esta aproximación al cálculo de los AVPP es la no comparabilidad con otras poblaciones que seguramente tienen esperanza de vida, diferentes. No se pueden comparar los AVPP entre dos o más situaciones a estudiar si el criterio de cálculo no es el mismo (OPS B. E., 2003).

\section{Mortalidad en Hospital San Felipe, 2014}

Se describen a continuación los principales hallazgos de la investigación en las variables procedencia del paciente, rangos de edad, causa de muerte según clasificación y tasa de muerte hospitalaria.

\subsection{Mortalidad hospitalaria según lugar de proce-} dencia, Hospital San Felipe, año 2014

El porcentaje más importante de defunciones proceden del departamento de Francisco Morazán, lo que se explica porque el hospital se encuentra en la ciudad de Tegucigalpa, que es la cabecera departamental y capital de la República de Honduras; ocupan el segundo lugar los departamentos de El Paraíso y de Comayagua que comparten el mismo porcentaje, los que se encuentran en el oriente y centro del país y los pacientes acceden al hospital por vías de acceso directo; en el tercer lugar, se encuentran los departamentos de Valle y Choluteca, que se encuentran en el sur del país y tienen vías de acceso directo y cercanos a Tegucigalpa (Cuadro1).

Cuadro 1: Hospital San Felipe: distribución de las defunciones según departamento de origen del paciente, año 2014

\begin{tabular}{|l|c|c|}
\hline \multicolumn{1}{|c|}{ Departamento } & Casos & Porcentaje \\
\hline Francisco Morazán & 84 & 70.5 \\
\hline El Paraíso & 7 & 5.04 \\
\hline Comayagua & 6 & 5.04 \\
\hline Santa Bárbara & 4 & 3.36 \\
\hline Valle & 4 & 3.36 \\
\hline Choluteca & 4 & 3.36 \\
\hline Cortés & 3 & 2.52 \\
\hline Colón & 2 & 1.68 \\
\hline Olancho & 2 & 1.68 \\
\hline Yoro & 2 & 1.68 \\
\hline Gracias a Dios & 1 & 0.84 \\
\hline Intibucá & 1 & 0.84 \\
\hline TOTAL & 120 & 100 \\
\hline
\end{tabular}

Fuente: Expedientes de la Unidad de Estadística. Año 2014.

4.2 Mortalidad hospitalaria según rangos de edad. Hospital San Felipe, año 2014

Entre los pacientes del Hospital San Felipe, la media de edad es de 55 años con 53 días para el grupo total de hombres y mujeres, con una desviación típica de 18.9 años para un total de 120 datos.

El cuadro muestra que la media de edad es de 55.53 años con moda de 62 años, mediana de 57.50 años y desviación estándar de 18.90 años, por lo que los datos reflejan que los pacientes se encontraban en la etapa de adultez intermedia. En cuanto a la desviación estándar muestra la variabilidad con respecto a la media señalada (Cuadro 2). 
Gráfico 1. Hospital San Felipe: media y desviación típica de la edad según mortalidad hospitalaria, año 2014

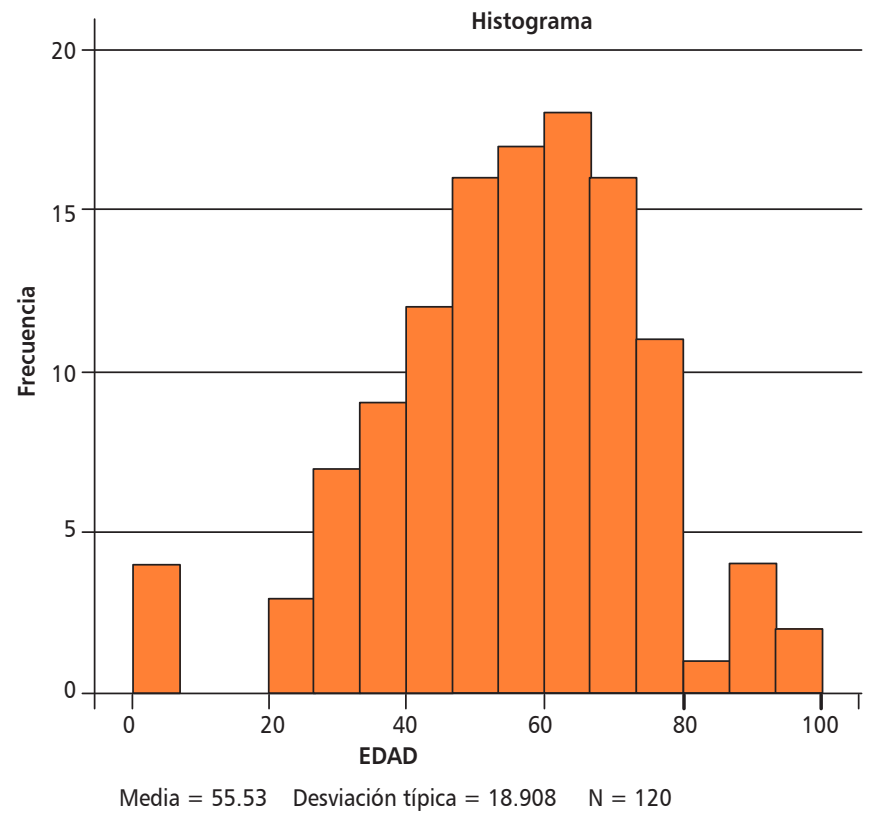

Fuente: Expedientes de pacientes. Unidad de Estadística. Hospital San Felipe.

Cuadro 2. Hospital San Felipe: estadísticos descriptivos de la variable edad, año 2014

\begin{tabular}{|l|c|}
\multicolumn{1}{|c|}{ Aspectos } & Valores \\
\hline Número de datos & 120 \\
\hline Rango & 95 \\
\hline Moda & 62 \\
\hline Media & 55.53 \\
\hline Mediana & 57.50 \\
\hline Desviación Estándar & 18.908 \\
\hline
\end{tabular}

Fuente: expedientes de pacientes. Unidad de Estadística de Hospital San Felipe.

Al agrupar las edades, el mayor porcentaje se encuentra en el grupo de 45 a 59 años ocupando el segundo lugar el grupo de 15 a 44 años y el de 71 años y más. El mayor porcentaje de muertes se ubica entre los rangos de 45 a 70 años con el $71.7 \%$ de los casos (Cuadro3).
Cuadro 3. Hospital San Felipe: mortalidad hospitalaria por grupos de edad, año 2014

\begin{tabular}{|l|c|c|}
\hline Grupo de edad & Casos & Porcentaje \\
\hline Menor de 1 año & 4 & 3.33 \\
\hline De 1-4 años & 0 & 0 \\
\hline De 5- 14 años & 0 & 0 \\
\hline De 15-44 años & 25 & 21 \\
\hline De 45 -59 años & 37 & 31.7 \\
\hline De 60 -70 años & 27 & 23 \\
\hline De 71 y más años & 25 & 21 \\
\hline TOTAL & 120 & 100 \\
\hline
\end{tabular}

Fuente: datos obtenidos de expedientes de pacientes. Unidad de Estadística de Hospital San Felipe.

4.3 Mortalidad hospitalaria según sexo y causa de muerte Hospital San Felipe, año 2014

Al desagregar los datos según sexo se identifica que el mayor porcentaje de muertes se encuentra en el grupo de mujeres con el 63 por ciento de las muertes y el grupo de hombres representa el 37 por ciento de las muertes en el período observado (Gráfico 2).

Gráfico 2. Hospital San Felipe: distribución de la mortalidad por sexo. Año 2014

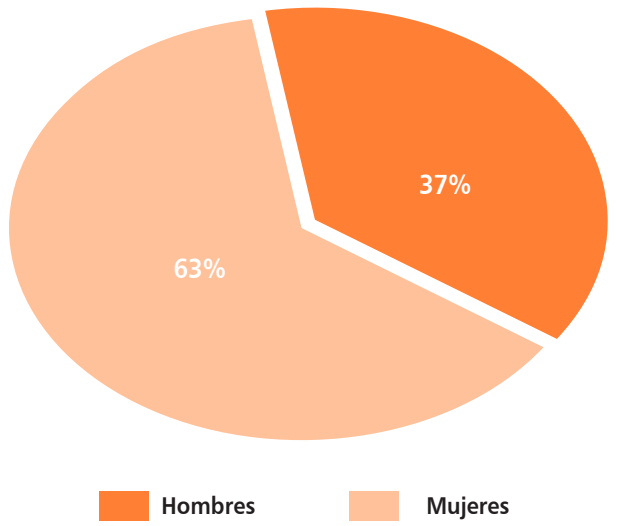

Fuente: Expedientes de la Unidad de Estadística. Año 2014. 
Se logra evidenciar que la causa básica de muerte más importante son los tumores $60.83 \%$, ocupando el segundo lugar $13.33 \%$, la septicemia y un tercer lugar las enfermedades del sistema circulatorio 7.5\% (Cuadro 4).

Cuadro 4. Hospital San Felipe: causa de mortalidad con clasificación internacional-10, Año 2014

\begin{tabular}{|l|c|c|c|}
\hline \multicolumn{1}{|c|}{ Causa } & CIE-10 & Casos & Porcentaje \\
\hline Tumores & C07X-C859 & 73 & 60.83 \\
\hline Septicemia & A19 & 16 & 13.33 \\
\hline Afecciones Perinatales & P051-P220 & 4 & 3.33 \\
\hline $\begin{array}{l}\text { Diabetes No insulinode- } \\
\text { pendiente }\end{array}$ & E110-E129 & 4 & 3.33 \\
\hline $\begin{array}{l}\text { Enfermedad del Sistema } \\
\text { Circulatorio }\end{array}$ & I00-I99 & 9 & 7.5 \\
\hline $\begin{array}{l}\text { Enfermedad del Sistema } \\
\text { Respiratorio }\end{array}$ & J00-J99 & 7 & 5.83 \\
\hline $\begin{array}{l}\text { Enfermedad del Sistema } \\
\text { Digestivo }\end{array}$ & K00-K93 & 7 & 5.83 \\
\hline Total & 120 & 100 \\
\hline
\end{tabular}

Fuente: datos de expedientes de Unidad de Estadística de Hospital San Felipe.

\subsection{Tasa de mortalidad hospitalaria, Hospital San}

\section{Felipe, año 2014}

La tasa bruta de mortalidad en el período observado es de $0.78(120 / 15,377)$ y expresa que del total de cien egresos, el riesgo de muerte es de menos del uno por ciento para el hospital en el período observado (Ver Cuadro 5).

Cuadro 5. Tasa de mortalidad bruta del Hospital San Felipe

\begin{tabular}{|l|c|c|c|}
\hline Servicio & Fallecidos & Egresos & Tasa (\%) \\
\hline Oncología & 72 & 1744 & 46.82 \\
\hline Medicina Interna & 40 & 980 & 26.01 \\
\hline Maternidad & 4 & 9763 & 02.60 \\
\hline Asilo de Inválidos & 4 & 7 & 02.60 \\
\hline Cirugías & 0 & 1401 & 0 \\
\hline Oftalmología & 0 & 527 & 0 \\
\hline Pediatría & 0 & 995 & 0 \\
\hline Total Hospital & 120 & 15377 & 0.78 \\
\hline
\end{tabular}

Fuente: Unidad de Estadística. Hospital San Felipe. 2014.

La tasa de mortalidad neta del Hospital San Felipe en el año 2014 es de 0.96 y expresa que del total de cien egresos (muertes) acaecidas a partir de ingresos realizados al hospital de más de 48 horas, el riesgo de muerte es de 1. A nivel de los servicios, el que presenta mayor tasa neta es el Asilo de Inválidos, ya que por tratarse de un asilo, la frecuencia de egresos en el mismo es poca y el comportamiento de este servicio, es diferente al del resto del hospital, ya que representa un proceso de permanencia a largo plazo. En relación a los otros servicios, el de oncología ocupa el primer lugar, presentando el mismo comportamiento el de medicina interna y el de maternidad en tercer lugar con menos del 1 por ciento.

\section{Cuadro 6. Tasa neta por servicio}

\begin{tabular}{|l|c|c|c|}
\multicolumn{1}{|c|}{ Servicio } & Fallecido & Egreso & Tasa (\%) \\
\hline Oncología & 72 & 1,744 & 4.12 \\
\hline Medicina Interna & 40 & 980 & 4.08 \\
\hline Maternidad & 4 & 9,763 & 0.04 \\
\hline Asilo De Inválidos & 4 & 7 & 57.14 \\
\hline Total & 120 & $12,494^{*}$ & 0.96 \\
\hline
\end{tabular}

Fuente: Unidad de Estadística de Hospital San Felipe. 2014

*Incluye únicamente las muertes de los servicios en los que se registraron muertes.

\section{Años de vida potencialmente perdidos to- mando como base la esperanza de vida hasta el año 2013}

La esperanza de vida es un indicador que resume las condiciones de mortalidad de una distribución amplia de personas con vidas que pueden ser de muy distinta duración. Representa el número promedio de los años que llegan a vivir una generación de personas que llegan a nacer en el mismo intervalo de tiempo. Es una medida general de la calidad de vida en un país y puede ser pensado como un indicador de la tasa de retorno potencial de la inversión en capital humano (http://celade.cepal. org/redatam/pryesp/cairo/WebHelp/Metalatina/esperanza_de_vida_al_nacer.htm).

En el caso del Hospital San Felipe en la casuística que se presenta, los tumores representan la primera causa de carga de enfermedad siendo el sexo femenino, el más afectado. La razón de muerte prematura hombre- mujer de 2 veces más en las mujeres que en los hombres.

La segunda causa de muerte es la septicemia y la razón hombre- mujeres por esta causa es similar en ambos sexos (Uno a uno). 
Año de vida potencialmente perdidos (AVPP) tomando como base la esperanza de vida hasta el año 2013

\begin{tabular}{|l|c|c|c|c|c|c|}
\hline \multicolumn{1}{|c|}{ Causa } & $\begin{array}{c}\text { Casos en } \\
\text { mujeres }\end{array}$ & $\begin{array}{c}\text { Esperanza de } \\
\text { Vida }\end{array}$ & AVPP & $\begin{array}{c}\text { Casos en } \\
\text { hombres }\end{array}$ & $\begin{array}{c}\text { Esperanza de } \\
\text { Vida }\end{array}$ \\
\hline Tumores & 45 & 76 & 1054 & 26 & 72 & 491 \\
\hline Septicemia & 8 & 76 & 98 & 6 & 72 & 93 \\
\hline Afecciones Perinatales & 3 & 76 & 227 & 1 & 72 & 72 \\
\hline Diabetes no insulinodependiente & 1 & 76 & 14 & 2 & 72 & 47 \\
\hline Enfermedad del Sistema Circulatorio & 4 & 76 & 66 & 3 & 72 & 33 \\
\hline Enfermedad del Sistema Respiratorio & 3 & 76 & 15 & 2 & 72 & 63 \\
\hline Enfermedad del Sistema Digestivo & 2 & 76 & 42 & 4 & 72 & 65 \\
\hline
\end{tabular}

Fuente: Unidad de Estadística de Hospital San Felipe. 2014

En las afecciones perinatales, la razón hombre- mujer es de 3 veces más en el sexo femenino que en el masculino.

En la diabetes no insulinodependiente, la razón hombre-mujer es de 3 veces más en los hombres que en las mujeres.

A nivel de las afecciones del sistema circulatorio, la razón hombre-mujer de 2 veces más en las mujeres que en los hombres.

En las enfermedades del sistema respiratorio, la razón hombre- mujer es de 4 veces más en los hombres que en las mujeres.

En las enfermedades del sistema digestivo, la razón hombre-mujer de 1.5 veces más en los hombres que en las mujeres.

\section{Reflexión final}

Honduras ha mejorado los datos de salud con respecto a la esperanza de vida, de tal forma que, en el año 2013 la esperanza de vida en Honduras subió hasta llegar a 73,80 años. Ese año, la esperanza de vida de las mujeres fue de 76,22 años mayor que la de los hombres, que fue de 71,51 años (72). Es decir, se encuentra en la parte media del ranking de países por esperanza de vida (http://www. datosmacro.com/demografia/esperanza-vida/honduras).

Aún con todos los esfuerzos que el país ha realizado en cuanto al proceso de mejora de las condiciones de salud, el estudio muestra que la carga de enfermedad producida por las enfermedades crónicas en la mortalidad de los pacientes hospitalizados que se atienden en el Hospital San Felipe, en el año 2014, es sumamente importante tomando el hecho de que se trata de un hospital nacional de referencia para todo el país, ya que es sede del Centro Nacional de Cáncer, del Centro Nacional de Rehabilitación y del Centro Nacional de Oftalmología, contiene a su vez una sala de maternidad de referencia nacional que atiende a pacientes catalogadas de bajo riesgo y una consulta ambulatoria de atención de medicina especializada y de medicina general. De acuerdo al sistema actual, actúa en la red sanitaria, como captor de los casos que las otras instituciones tanto del sector público como del privado, envían a los diferentes centros de referencia señalados.

Los datos denotan la importancia del acceso geográfico a la atención del mismo de tal forma que más de la mitad de las defunciones corresponden al departamento de Francisco Morazán, en cuya cabecera departamental, se encuentra ubicado el hospital.

Todos los países de América Latina, incluyendo a Honduras, se encuentran afrontando un doble desafío, seguir disminuyendo las enfermedades de tipo infecciosa y parasitaria y abordar la creciente mortalidad por enfermedades no transmisibles. Las implicaciones en las acciones de políticas públicas de salud son muchas, por un lado, no es posible dejar de invertir en mejorar la salud de los niños, también es más que probable que por el creciente crecimiento de los adultos mayores se exijan acciones de diversa índole en términos de prevención, curación y cuidados (Di Cesare, 2011).

Los grupos de edad más afectada para ambos sexos, es el que se encuentra en edad productiva destacándose como causa de muerte, los tumores, la diabetes, las enfermedades del aparato circulatorio, respiratorio y 
digestivo y apareciendo en menor proporción, las enfermedades perinatales. La carga de enfermedades crónicas tiene serios efectos adversos en la calidad de vida de los individuos afectados, es causa de muertes prematuras, tiene efectos económicos importantes y subestimados en las familias, las comunidades y las sociedades en general (OMS, 2005).

En las mujeres las primeras causas de AVPP, se encuentran en los tumores, las septicemias, las enfermedades perinatales y las enfermedades del sistema circulatorio. En los hombres, fue la diabetes no insulinodependiente, las enfermedades del sistema respiratorio y del sistema digestivo. En el caso del sexo femenino las principales causas de muerte analizadas aportaron la mayor cantidad de AVPP (1516 de las mujeres y 864 de los hombres).

Es importante destacar que en las diferencias de género, las neoplasias juegan un rol clave para el perfil de mortalidad de las mujeres adultas, mucho más de lo que se observa en el caso de los hombres (Di Cesare, 2011).

De acuerdo a datos suministrados por la CEPAL, al analizar la mortalidad en América Latina, en el caso de las mujeres, las causas más importantes de muerte se encuentran en las neoplasias y las enfermedades del aparato circulatorio (CEPAL, 2007), los datos que surgen de esta investigación están acordes con esta aseveración, sobre todo por el hecho de que las neoplasias en el grupo de mujeres evidenciado en este trabajo están relacionados con cáncer cervicouterino, por lo que la Organización Mundial de la Salud señala que: “... muchos países en desarrollo siguen viéndose afectados desproporcionadamente por la doble carga que supone la alta tasa de cánceres provocados por infecciones (como el cáncer cervicouterino)..." (http://www.who.int/mediacentre/ news/releases/2014/cancer-report-20140203/es/) , razón por la cual, las acciones de prevención deben duplicarse a nivel de micro, meso y macrogestión elaborando y operacionalizando políticas que trasciendan los datos presentados.

La septicemia, es la segunda causa de carga de enfermedad en esta serie y en los últimos 30 años su morbi mortalidad no ha variado, incluso hay autores que insisten en afirmar que se ha incrementado a un ritmo de 7 a por ciento anual por diversos factores como son el aumento de expectativa de vida que deriva en un mayor número de procesos crónicos en la población, incremento en la realización de técnicas invasivas, estados de inmunosupresión por fármacos, enfermos tratados con quimioterapia, hace que el número de fallecimientos por esta razón se eleve e importantes estudios epidemiológicos han dejado clara la evidencia de que es responsable de más muertes que el infarto agudo de miocardio o el ictus (Gonzáles, 2003). De acuerdo a los datos presentados, los factores enunciados se encuentran en los pacientes que fallecieron en el Hospital San Felipe.

Una mejor atención sanitaria, la detección temprana y el tratamiento oportuno son una alternativa eficaz para reducir las enfermedades no transmisibles. Muchas intervenciones sanitarias se consideran costo eficaces especialmente en comparación con los costosos procedimientos que suelen requerirse cuando la detección y el tratamiento se han retrasado o llegan en etapa avanzada de la enfermedad. Es preciso seguir fortaleciendo los sistemas de salud para ofrecer un conjunto eficaz, realista y asequible de intervenciones y servicios para las personas afectadas (OMS, Informe sobre la situación mundial de las enfermedades no transmisibles 2010. Resumen de orientación, 2010).

Dado que la septicemia intrahospitalaria es una complicación en la que se conjugan diversos factores de riesgo que son susceptibles de prevención y control, las instituciones de salud deben establecer mecanismos eficientes de intervención que permitan la aplicación de medidas preventivas y correctivas encaminadas a la disminución de los factores de riesgo que inciden en la distribución y frecuencia de estas infecciones (Salud, 2010).

En cuanto a las afecciones perinatales, se ha establecido que la mortalidad por esta causa depende de varios factores que no están confinados exclusivamente al área médica hospitalaria. La comunidad tiene un papel muy importante, se deben realizar programas de educación en general y en salud reproductiva, la descentralización de los sistemas de salud y asegurar una comunicación ade- 
cuada entre los diferentes niveles y centros de atención médica, así como el fortalecimiento del cuidado prenatal a través de personal calificado y una mejor infraestructura (Murguía de Sierra, Lozano, \& Santos, 2005).

Desde que en el año de 1976, Rutsteinse preguntara al observar los datos de mortalidad de un hospital ante una muerte: " $i$... por qué sucedió?..." se mantiene la necesidad de conocer las causas que desencadenaron la muerte en enfermedades que no debieran terminar fatalmente en ese momento. Analizar las características clínicas de los fallecidos así como las causas de muerte, permiten conocer no solo la estadística de mortalidad hospitalaria sino también lo más importante, la evolución de los datos y las medidas correctivas que se tienen que realizar así como su tendencia con el tiempo. Si es importante establecer el nivel de complejidad porque la afluencia de internados dependerá del peso de la composición de los distintos servicios en la institución, entre los que habrá algunos con mayor tecnología o profesionales con mayor valoración por parte de la población o por ser centro de valoración de determinada enfermedad (Miceli, 2013). Bajo este contexto, el Hospital San Felipe cumple con los criterios de ser una institución de referencia nacional y albergar en su interior al centro nacional de referencia oncológica del país.

\section{BIBLIOGRAFÍA}

- Arriaga, E. (1994). Los años de vida perdidos: su utillización para medir el nivel y cambio de la mortalidad.

- CEPAL. (2007). La mortalidad en América Latina: una trayectoria auspiciosa pero heterogénea.

- Cuba, M.d. (2010). Indicadores Básicos para el análisis del Estado de Salud de la Población. Habana.

- Demograficos/, h.-h.-c. (s.f.).

- Di Cesare, M. (. (2011). El perfil epidemiologico de América Latina y el Caribe: desafíos, límites y acciones. Santiago de Chile.

- Gonzáles, M. E. (2003). Relaciones entre mortalidad hospitalaria y sepsis durante 15 años. La Habana.

- http://celade.cepal.org/redatam/pryesp/cairo/We-
bHelp/Metalatina/esperanza_de_vida_al_nacer.htm. (s.f.).

- http://datos.bancomundial.org/indicador/SP.DYN. LEO0.IN/countries. (s.f.).

- http://www.datosmacro.com/demografia/esperanzavida/honduras. (s.f.).

- http://www.who.int/mediacentre/news/releases/2014/ cancer-report-20140203/es/. (s.f.).

- INE, D. s. (2014). Mujeres y hombres en España 2014.

- Marlow, A. (1995). Potential years of life: what ies demonimator? Journal of Epidemiology and Community Health. 1995; 49: 320-322.

- Martínez Sánchez, J., Ramos Aceitero, J., Alvarez Díaz, M., \& Rojas Fernández, C. (2013). Cálculo de Años potenciales de vida perdidos (APVP) en Extremadura. Comparación de cuatro métodos .

- México.CEMECE, C. c. (2009). Material de consulta. Actualizaciones de CIE-10. México, D.F.

- Miceli, I. (2013). Análisis de la mortalidad hospitalaria. Editorial. Buenos Aíres: Medicina-Volumen 73.No. 2. 2013.

- Murguía de Sierra, M. T., Lozano, R., \& Santos, J. I. (2005). Mortalidad perinatal por asfixia en México: problema prioritario de salud pública por resolver. México, D.F.: Boletín Médico del Hospital Infantil de México.

- OMS. (2005). Prevención de las enfermedades crónicas: una inversión vital.

- OMS. (2010). Informe sobre la situación mundial de las enfermedades no transmisibles 2010. Resumen de orientación. Ginebra.

- OPS. (1986). Años de vida potencial perdidos. Boletín epidemiológico. Boletín epidemiológico. 5(5): 3-6.

- OPS, B. E. (2003). Técnicas para la medición dek impacto de la mortalidad: Años potenciales de vida perdidos. Washington: Vol 24.No.2.

- Salud, S. D. (2010). Metodología para anáisis de mortalidad asociada a infección intrahospitalaria. Bogotá.

- Velásquez V, A., USAID/PERÚ, \& PRAES. (2006). Guía metodológica para las estimaciones epidemiológicas del estudio de carga de enfermedad. USAID/Perú. 